\title{
Effects of stimulus probability and information feedback on response biases in children's recognition memory
}

\author{
DANIEL B. BERCH \\ Morehead State University, Morehead, Kentucky 40351
}

\begin{abstract}
Stimulus probability and amount of information feedback were varied factorially to determine their effects on kindergartners' response biases in a continuous recognition memory task. The results showed that when subjects received trial-by-trial feedback, there was a direct relationship between the tendency to respond "old" and the proportion of items that were in fact old. However, when no information was given about stimulus probabilities, the bias for reporting "old" varied inversely with the proportion of items that was actually old. It was also determined that this latter type of bias was at least partially due to the occurrence of first-order sequential stimulus contrast; that is, the tendency to respond "old" was found to be greater when the stimulus item on the preceding trial was new than when it was old.
\end{abstract}

What are the primary factors affecting the nature of decision processes in children's recognition memory? Actually, very little is known about this, since "response bias" is typically viewed as an extraneous variable that should be nullified either experimentally (through the use of the 2-AFC paradigm) or statistically (e.g., correction for guessing). Nevertheless, a thorough understanding of item recognition cannot be achieved without an adequate knowledge of response biases and their controlling factors. Therefore, the present experiment was designed to provide some much needed data regarding the decision-based component of children's recognition performance.

The first question posed was: To what extent, if any, do children set their criterion so as to optimize correct responding? The most direct way to determine this would be to vary the a priori probability, that is, the proportion of items that are actually old (hereafter referred to as "stimulus probability" or q). Under such conditions, subjects could maximize correct recognitions and minimize false recognitions by adopting an increasingly liberal criterion as the value of $q$ increased. In the psychophysical literature, this type of response bias is commonly referred to as "probability assimilation." Of course, assessing children's assimilation tendencies in this manner would require that they "know" the stimulus probabilities being used. Adult subjects are usually informed of the value of $q$ by explicit instruc-

This study was supported by a grant from the Research Allocations Committee of the University of New Mexico. The author is grateful to the administrative and teaching staffs of the Children's Garden Center and the Albuquerque Preschool and Kindergarten Co-operative for providing subjects for this research. Thanks are also extended to Sheldon Benson for his assistance in data collection. Requests for reprints should be sent to Daniel B. Berch, Department of Psychology, UPO 752, Morehead State University, Morehead, Kentucky 40351. tions. However, since it is unlikely that young children would fully comprehend (and remember as well) instructions about relative proportions (especially for stimulus probabilities other than .50), it was decided that information about $q$ would have to be presented by some other means. The most obvious candidate was trial-by-trial feedback. Although this technique had not been previously used in studies of children's recognition memory, findings from probability learning experiments suggested that feedback is indeed an effective method for conveying probabilistic information to young children (Messick \& Solley, 1957).

A second, yet equally important, concern of the present research was to determine how manipulation of stimulus probabilities affects response biases when children are not informed of the value of q. Actually, in most recognition memory studies with young children, no stimulus information whatever is provided. Under such conditions, there is no standard that subjects can use as a basis for establishing an assimilation bias. The only clue as to what sort of bias might develop under these conditions comes from a study with college students. Using q values of .20 and .50 in a continuous recognition task, Marken and Sandusky (1974) found that providing no stimulus information led to the adoption of a more liberal criterion by the .20 group than by the .50 group. In the psychophysical literature this is referred to as "probability contrast," a type of bias consisting of an inverse relationship between the value of q and the tendency to respond "old." Interestingly, Marken and Sandusky also discovered that the contrast bias in their study could at least partially be accounted for by the occurrence of first-order sequential stimulus contrast. That is, the bias for reporting "old" was found to be greater when the stimulus on the preceding trial was new than when it was old.

The findings of Marken and Sandusky (1974) led the 
present writer (Berch, 1976) to reanalyze the data from a previous study of children's recognition memory (Berch \& Evans, 1973) in order to determine whether there was any evidence of sequential dependencies. This analysis did in fact reveal the existence of sequential stimulus contrast in the performance of both kindergartners and third graders. However, since only one value of $\mathrm{q}(.50)$ was employed in the Berch and Evans study, there was no way of ascertaining if these children had also adopted an overall, probability contrast type of bias. Therefore, two values of $q$ were utilized in the present experiment, enabling a direct assessment of whether young children will exhibit probability contrast when no stimulus information is provided.

\section{METHOD}

\section{Subjects}

The subjects were 40 kindergartners from private schools in Albuquerque, New Mexico. They ranged in age from 5 years, 0 months to 6 years, 5 months, with a mean age of 5 years, 8 months.

\section{Design}

The basic design of the study was a 2 by 2 factorial, with stimulus probability $(.25, .50)$ and feedback condition [feedback (FB), no feedback (NFB)] as between-subjects variables. Ten subjects were randomly assigned to each of the four resulting treatment conditions: .25-FB, .50-FB, .25-NFB, and .50-NFB.

\section{Materials}

The pretraining materials consisted of pictures of common objects, such as chair, shirt, book, etc. For the .50 conditions, the deck contained nine different pictures, each one appearing twice. The pretraining deck for the .25 conditions contained 18 different pictures, 6 of which appeared twice and 12 once. Thus, the pretraining decks were constructed so as to match the stimulus probability that would subsequently be reflected in the materials of the main experimental task. In other words, the deck for the .50 conditions included nine, or $50 \%$, old items, and the .25 deck included six, or $25 \%$, old items.

The materials for the main task consisted of $724 \times 6$ in. cards, each containing a two-digit number drawn in black. Repeated digits, such as 33 , were excluded. For the .50 conditions, there were 36 two-digit numbers, each one appearing twice in the deck. Three repetition lags of 0,6 , or 13 cards were used, where lag refers to the number of cards intervening between the first and second presentations of an item. The 72 cards were arranged in three blocks of 24 such that each block contained 12 new items (appearing for the first time) and 12 old items (appearing for the second time). Within each block, four twodigit numbers were randomly assigned to each of the three lags. Next, the order of occurrence of the four stimuli associated with each lag was randomized. Thus, all blocks were comparable in that they contained the same number of old and new items as well as the same number of stimuli at each of the different lags. Furthermore, the blocks were constructed so that there were no more than four runs of old or new items. Finally, the order of occurrence of the blocks was counterbalanced.

For the .25 conditions, there were 54 two-digit numbers, 18 appearing twice in the deck and 36 appearing once. Thus, the response of "old" would be correct $25 \%$ of the time (18 out of $72)$. As with the .50 conditions, three repetition lags of 0,6 , or 13 cards were used. The 72 cards were arranged in three blocks of 24 such that each block contained 18 new items and 6 old items. Randomization and counterbalancing were carried out in exactly the same manner as for the .50 conditions.

\section{Procedure}

All subjects were given a series of pretraining trials, consisting of pictures of common objects presented one at a time. Each child was instructed that on every trial he was to say "old" if he remembered having seen that item earlier in the deck, or "new" if he did not. Although trial-by-trial feedback was not given, all subjects heard random comments such as "You're doing very well" or "You're pretty good at this game." Immediately following pretraining, all children were told that they were going to play another, similar game. The experimenter then presented the two-digit numbers one at a time, pronouncing each number as single digits (e.g., "19" was pronounced as "one-nine"). Two of the treatment groups received trial-by-trial feedback and two did not. Feedback consisted of the experimenter stating the correct response ("old" or "new") immediately following the child's response on every trial. ${ }^{1}$

\section{RESULTS}

Memory-operating-characteristic (MOC) plots of the mean hit rates and false-alarm rates for each treatment group are shown in Figure 1. It can be readily observed that the point for Group .50-FB is above and to the right of the point for Group .25-FB, indicating probability assimilation, that is, a direct relationship between the probability of occurrence of an "old" stimulus and the bias for reporting "old." However, for the NFB conditions, the point for the .25 group is above and to the right of that of the .50 group. This result reflects a probability contrast type of bias, that is, an inverse relationship between stimulus probability and the tendency to report "old." To evaluate these results statistically, a nonparametric index of response bias

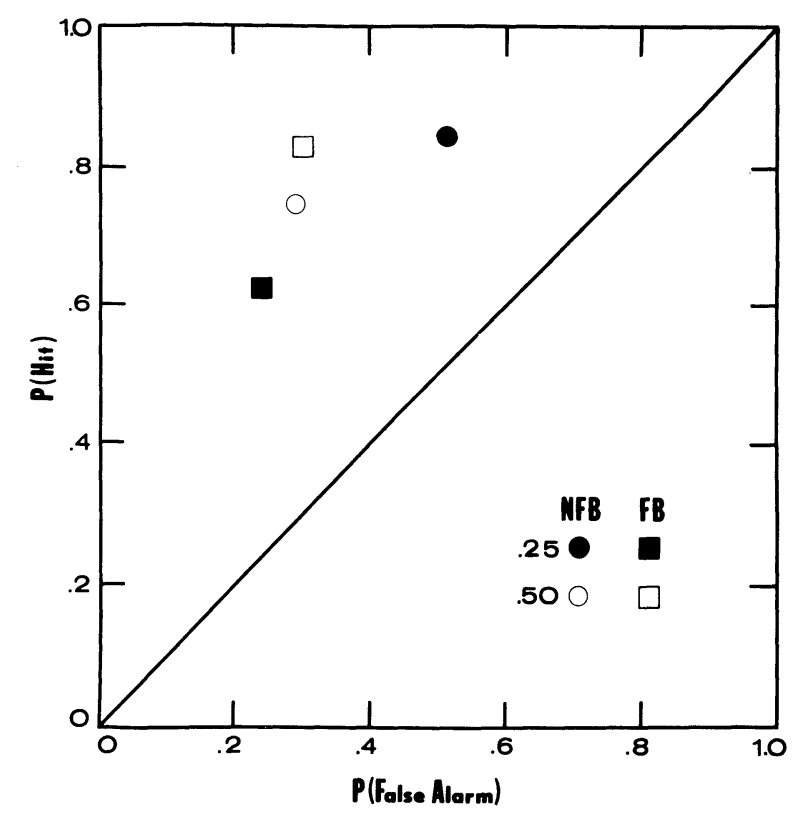

Figure 1. MOC plots of the mean hit rates and false-alarm rates for the four treatment conditions. 
(RI) was used as the dependent variable (Frey \& Colliver, 1973). ${ }^{2}$ First, a separate RI score was computed for each subject. Then a 2 (stimulus probability) by 2 (feedback condition) factorial analysis of variance was performed on the square-root transformations $(\sqrt{x+1.5})$ of the RI scores. Although the main effects were nonsignificant, the interaction was highly significant $[F(1,36)=15.03, p<.001]$, thus providing statistical support for the differential bias effects observed in Figure 1.

To determine whether sequential stimulus contrast was at least partially responsible for the overall contrast bias exhibited by the NFB groups, first-order sequential dependencies were computed for each subject in the .25-NFB and .50-NFB conditions using the following formula:

$$
\begin{aligned}
\text { "Old" bias }= & P\left(\mathrm{Hit}_{\mathrm{T}} \mathrm{O}_{\mathrm{n}-1}\right)+\mathrm{P}\left(\mathrm{FA} / \mathrm{O}_{\mathrm{n}-1}\right) \\
& -\mathrm{P}\left(\mathrm{Hit} / \mathrm{N}_{\mathrm{n}-1}\right)-\mathrm{P}\left(\mathrm{FA} / \mathrm{N}_{\mathrm{n}-1}\right),
\end{aligned}
$$

where $\mathrm{O}_{\mathrm{n}-1}$ and $\mathrm{N}_{\mathrm{n}-1}$ stand for an "old" and a "new" stimulus on the previous trial, respectively. Negative scores resulted for 8 out of 10 subjects in each group, indicating that sequential effects did occur.

\section{DISCUSSION}

The results of this study demonstrate clearly that the amount of stimulus information conveyed to young children strongly influences the nature of the response biases they will adopt in the context of a continuous recognition memory task. Unfortunately, the clarity of the empirical data does not extend to the theoretical domain. Recently, Dusoir (1975) suggested that, in order to accommodate the results of experiments such as the present one (similar findings have occurred in psychophysical studies of signal recognition with adult subjects, e.g., Tanner, Rauk, \& Atkinson, 1970), a model must allow the probability of reporting "old" to increase or decrease as q increases, depending on the information conditions. Following a critical examination of extant treatments of bias (Creelman \& Donaldson, 1968; Healy \& Jones, 1973; Parks, 1966; Thomas \& Legge, 1970), Dusoir concluded that all of these models totally fail in this regard.

Despite the lack of an adequate theoretical framework for the present results, it is hoped that this experiment will provide a sound basis for continued investigation into the role of decision processes in children's recognition performance.

\section{REFERENCES}

BERCH, D. B. Criterion change in continuous recognition memory: A sequential effect. Bulletin of the Psychonomic Society, 1976, 7. $309-312$.
Berch, D. B.. \& Evans, R. C. Decision processes in children's recognition memory. Journal of Experimental Child Psychology, 1973, 16. 148-164.

Brown, A. L., \& Scotr, M. S. Recognition memory for pictures in preschool children. Journal of Experimental Child Psychology, 1971, 11, 401-412.

Creelman, C. D., \& Donaldson, W. ROC curves for discrimination of linear extent. Journal of Experimental Psychology, 1968, 77, 514-516.

Dusorr. A. E. Treatment of bias in detection and recognition models: A review. Perception \& Psychophysics, 1975, 17 167-178.

Frey, P. W.. \& Colliver, J. A. Sensitivity and responsivity measures for discrimination learning. Learning and Motivation, 1973, 4. 327-342.

Healy, A. F., \& Jones, C. Criterion shifts in recall. Psychological Bulletin, 1973, 79, 335-340.

PARKs, T. E. Signal-detectability theory of recognition-memory performance. Psychological Review, 1966, 73, 44-58.

MARKen. R. S., \& SANdusKy, A. J. Stimulus probability and sequential effect in recognition memory. Bulletin of the Psychonomic Society, 1974, 4, 49-51.

Messick. S. J..\& Solley, C. M. Probability learning in children: Some exploratory studies. Journal of Genetic Psychology, 1957, 90. 23-32.

Tanner, T. A.. JR., Rauk, J. A., \& Atkinson, R. C. Signal recognition as influenced by information feedback. Journal of Mathematical Psychology, 1970, 7, 259-274.

Thomas, E. A. C., \& Legge, D. Probability matching as a basis for detection and recognition decisions. Psychological Review, 1970, 77. 65-72.

\section{NOTES}

1. Brown and Scott (1971) found that children preferred saying "yes" or "no" rather than "old" or "new." Although I also found this tendency to some extent in pretraining, I had to require responses in the form of "old" or "new" because it would have been too confusing if subjects in the feedback groups had to respond "yes" or "no" and then receive feedback stated in the same terms.

2. Although RI was originally devised for use in the discrimination learning paradigm, the fact that it reflects a responsivity or guessing type of bias rather than a perceptual bias makes it particularly appropriate for use in the recognition memory paradigm. This nonparametric index is based on the assumption that neutral bias is reflected by points falling on the negative diagonal of the unit square and that positive (liberal) bias is proportional to the distance of the data point above this diagonal, while negative (conservative) bias is proportional to the distance below it. Although RI can be obtained graphically from the unit square, Frey and Colliver (1973) have derived the following formula which permits the computation of this index in terms of hit rate (HR) and false-alarm rate (FAR) values:

$$
R I=\frac{H R+F A R-1}{1-(H R-F A R)^{2}} .
$$

(Received for publication June 22, 1977.) 